\title{
Influence of the Lewis basicity hardness of recrystallization solvents on the coordination sphere of the complex [Co(3,5-dinitrobenzoate-O,O')2]
}

\author{
Andrés F. Pérez, Daniela Fonseca, John J. Hurtado, Mario A. Macías \\ Chemistry Department, Universidad de los Andes, Carrera 1 No. 18-12, Bogotá, Colombia \\ af.perez10@uniandes.edu.co/ma.maciasl@uniandes.edu.co
}

Coordination compounds are obtained by the reaction between Lewis acids (metal) and Lewis bases (ligand). According to Pearson's acid-base theory, also known as HSAB theory, the stability of the compound depends on the hardness of the acid and the base and their affinity. Hard bases tend to react with hard acids and soft bases prefer to react with soft acids. By just varying the hardness of the metal, the ligand or the solvent it is possible to substantially change the structure of the complex, giving a plethora of possibilities for the synthesis of different coordination compounds. This phenomenon was evidenced in coordination compounds of $\mathrm{Cu}$ (II) and $\mathrm{Zn}$ (II), where changing the ligand from 3,5-dinitrobenzoate to pyrazole derivatives affects the number of metallic centers present after crystallization ${ }^{1}$. Interested in these results we decided to study the effect of the recrystallization solvent on the coordination sphere of the complex $\left[\mathrm{Co}(3,5 \text {-dinitrobenzoate-O,O' })_{2}\right]$. Depending on the donor capabilities of the solvent, the complex undergoes a change on its coordination sphere, changing from a trinuclear Co (II) complex when the solvent is a soft base to mononuclear Co (II) complex when the solvent is a hard base. These structural changes are of great interest because materials and molecules that include cobalt in their structure have several spin states, which gives it interesting magnetic properties ${ }^{2}$.
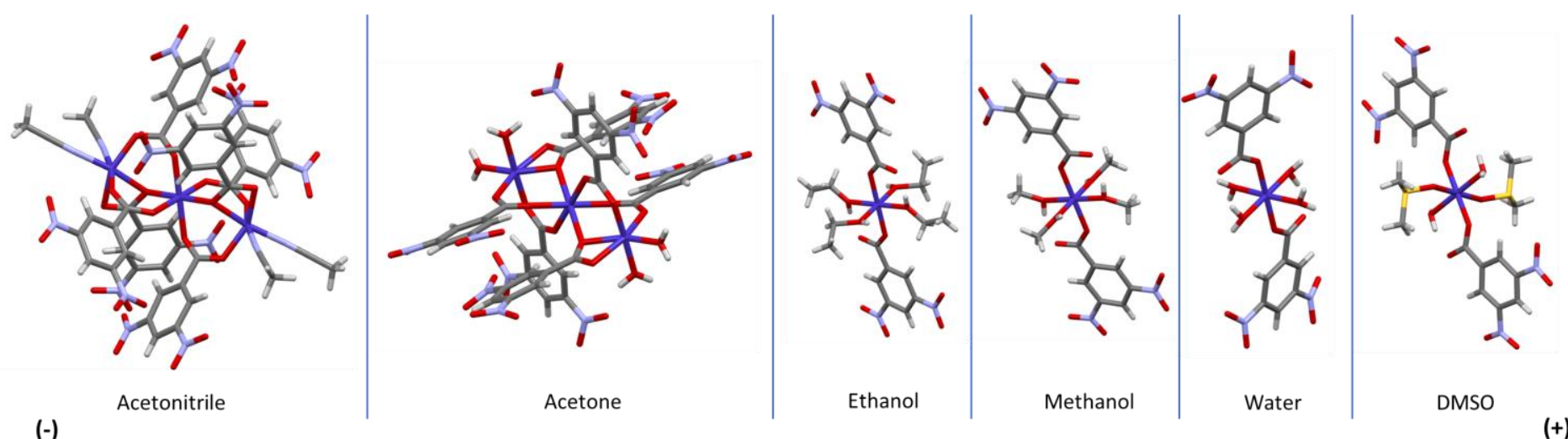

Lewis basicity

Figure 1. Effect of the solvent Lewis basicity on the chemical structure of the complex.

[1] Torres, J. F.; Macías, M. A.; Franco-Ulloa, S.; Miscione, G. P.; Cobo, J.; Hurtado, J. J. Cu(II) and Zn(II) Complexes with Dinitrobenzoates and Pyrazolyl Ligands: Structural and Thermal Stability Influence of N-H Moiety. Cryst. Growth Des. 2019, 19 (6), 3348-3357.

[2] Pham, A. T.; Abbasi, P.; Monache, G. D.; Mazarakioti, E. C.; Rawson, J. M.; Stamatatos, T. C.; Pilkington, M. Magneto-Structural Studies of Two M-O-M Bridged Homochiral Mixed Valence Co(II)/Co(III) Complexes. Polyhedron 2019, 170, 34-40.

Keywords: Lewis basicity; HSAB theory; coordination sphere; trinuclear complex; mononuclear complex

The authors thank the Universidad de los Andes, Science Faculty and Chemistry Department for providing funding (project Nos. INV2018-50-1354 and INV-2017-65-1683). M. A. M. thanks the 'Fondo de Apoyo para Profesores Asistentes' of the Facultad de Ciencias, Universidad de los Andes, Bogotá, FAPA-P18.160422.043. 